\title{
Reintroduction failure after undergoing oral immunotherapy or oral food challenges with cooked egg
}

\author{
Paula Cabrera-Freitag ${ }^{1}$, Alberto Alvarez-Perea ${ }^{1}$, Maria Jose Goikoetxea ${ }^{2}$, Sonsoles \\ Infante $^{3}$, Victoria Fuentes-Aparicio ${ }^{3}$, and Carmen D'Amelio ${ }^{2}$ \\ ${ }^{1}$ Hospital General Universitario Gregorio Marañón \\ ${ }^{2}$ Clínica Universidad de Navarra \\ ${ }^{3}$ Hospital Materno-Infantil Gregorio Marañón
}

September 28, 2021

\section{Reintroduction failure after undergoing oral immunotherapy or oral food challenges with cooked egg}

To the editor,

Food allergy has increased in recent decades currently affecting almost $6 \%$ of the european pediatric population (1). Egg is the second leading cause in food allergy (1) and in anaphylactic food reactions (2) among preschool-aged children in Europe. Recent studies have suggested a persistent tendency for egg allergy, estimating that less than $50 \%$ of these children can tolerate it by 2 years of age (3). However, up to $70 \%$ of egg-allergic children tolerate cooked egg (4). Some studies suggest that regular consumption of cooked egg in these children might increase their probability of tolerating egg in any presentation in the future (5, $6)$. In the past decades, complete egg avoidance was the recommended treatment in egg allergic patients. This recommendation has an important impact in quality of life of the patients and their families and does not guarantee the prevention of severe reactions due to accidental ingestion of even small amounts of the offending food $(2,7)$. Oral immunotherapy (OIT) for persistent egg allergy has emerged as an alternative therapy to egg avoidance. Tolerance can be induced by the administration of different egg products and maintained by the regular ingestion of egg several times per week, indefinitely.

Previous retrospectives studies had shown, that around $25 \%$ of children with negative oral food challenges OFC) did not reintroduce the food at home with a lower successful introduction rate for peanut $(32-60 \%)$ than for milk (10-22\%) or egg (13-29\%) (8-10). Aversion and refusal of the food, reactions at home after being tolerated in hospital and fear of reactions were the most reported reasons for peanut reintroduction failure (8-10).

As the type of food significantly influences the rate of non-adherence to its regular consumption (9), its causes might be also different depending on the food involved. The aim of our study was to analyze the leading causes of lack of adherence to the regular ingestion of cooked egg after undergoing OIT or an OFC with cooked egg.

Egg allergic patients among 2-17 years old, not following the recommendation of maintaining the intake of three eggs per week after undergoing OIT or OFC with cooked egg, were prospectively recruited from the Allergy Service of Hospital General Universitario Gregorio Marañón (Madrid) and Clínica Universidad de Navarra (Pamplona) between January 2019 and May 2020. The patients or their legal representatives, in the case of children under 12 years, fulfilled a questionnaire (supplemental file) regarding demographic and clinical information and the causes of lack of adherence or abandonment of regular intake of cooked egg. The questionnaire was also published in Twitter (Twitter Inc, San Francisco, Calif) and anonymously fulfilled. 
Qualitative variables were expressed in percentages and quantitative variables were expressed as median and interquartile range (IQR). The study was approved by the Ethics Committee for Investigation of both participating hospitals.

Twenty patients were included in the study [55\% female; median age: 10.7 years (IQR: 9-16.3)]. Fifteen (75\%) were recommended to maintain regular intake of cooked egg after OIT and $5(20 \%)$ after OFC. Patient's diagnosis of egg allergy was performed by a median age of 14.5 months (IQR: 11-16 months). Median time of egg avoidance before OFC or OIT with cooked egg was 6.1 years (IQR: 3.6-9.4 years).

Four (20\%) patients referred a frequency intake of two cooked eggs weekly, seven (35\%) referred the ingestion of one egg weekly and nine (45\%) only consumed breaded/floured foods with egg. The causes of lack of adherence to the regular intake of cooked egg are summarized in the figure. Most of the patients reported poor compliance as they did not like the taste (85\%), the texture (70\%) or the smell $(65 \%)$ of egg. Other causes of lack of adherence were being tired of eating the food in the same cooking presentation (55\%), the difficulty to find a variety of recipes (50\%), reactions at home after tolerance assessment by OFC or OIT (35\%), considering the recommended intake excessively frequent (25\%), fear of having a reaction (20\%), forgetting $(20 \%)$ and rejection of the appearance of the egg (10\%). One out of 20 patients (5\%) reported lack of adherence due to interference with other activities such as sports, other food allergies and living with egg allergic relatives.

According to our results, the most frequent cause for which patients decreased or stopped the regular ingestion of cooked egg is aversion of the food (related to the organoleptic characteristics of the egg: taste, texture, and/or smell) which is in accordance with other studies performed with other allergens $(9,10)$. The second cause of poor adherence in our study, was the lack of variety in their cooking preparation which has not been observed in other studies with other foods.

While in the case of peanuts, fear of having a reaction is among the first three causes of reintroduction failure (10), this does not seem to be an important cause in the case of egg, being the fifth cause referred by the patients in our study. We therefore believe that it is important to analyze the causes of lack of adherence to regular consumption of a food after having verified its tolerance, depending on the food and the type of presentation in which it is indicated to consume since they seem different and therefore the possible measures on which to act.

Our study has some limitations, as the relative small sample group. Time spam between food challenge and completion of the questionnaire is wide and probably those children fulfilling the questionnaire closer to the challenge, did it more correctly. Moreover, we do not have data on the exact timing of failure. The questionnaire is not validated, thus we cannot rule out that the content and way of asking may have influenced the results of our study. In addition, the inclusion criteria in those patients who fullfilled the questionnaire via Twitter could not be verified.

In our opinion, strategies to promote regular cooked egg consumption such as providing a variety of recipes for safe consumption with masked egg, suggesting practical recommendations to favor the regular intake or emphasizing in patient education regarding the importance of maintaining the regular consumption of the food could be useful to facilitate compliance in these patients and maintain the acquired tolerance.

\section{Key messages statement}

The causes of lack of adherence to regular consumption of a food after having verified its tolerance, seem to be different depending on the food and the type of presentation in which it is indicated to consume and therefore the possible measures on which to act.

Our study shows that the two most frequent causes for which patients decreased or stopped the regular ingestion of cooked egg after undergoing OIT or OFC with cooked egg, is aversion of the food (related to the organoleptic characteristics of the egg: taste, texture, and/or smell) and the lack of variety in their cooking preparation. 
Strategies to promote regular cooked egg consumption such as providing a variety of recipes for safe consumption with masked egg, could be useful to facilitate compliance in these patients and maintain the acquired tolerance.

\section{References}

1. Nwaru BI, Hickstein L, Panesar SS, Roberts G, Muraro A, Sheikh A; EAACI Food Allergy and Anaphylaxis Guidelines Group Prevalence of common food allergies in Europe: a systematic review and meta-analysis. Allergy. 2014 Aug;69(8):992-1007.

2. Grabenhenrich LB, Dölle S, Moneret-Vautrin A, Köhli A, Lange L, Spindler T, Ruëff F, Nemat K, Maris I, Roumpedaki E, Scherer K, Ott H, Reese T, Mustakov T, Lang R, Fernandez-Rivas M, Kowalski ML, Bilò MB, Hourihane JO, Papadopoulos NG, Beyer K, Muraro A, Worm M. Anaphylaxis in children and adolescents: The European Anaphylaxis Registry. J Allergy Clin Immunol. 2016 Apr;137(4):11281137.e1.

3. Xepapadaki P, Fiocchi A, Grabenhenrich L et al. Incidence and natural history of hen's egg allergy in the first 2 years of life-the EuroPrevall birth cohort study. Allergy 2016; 71(3):350-7.

4. Turner PJ, Mehr S, Joshi P, Tan J, Wong M, Kakakios A, Campbell DE. Safety of food challenges to extensively heated egg in egg-allergic children: a prospective cohort study. Pediatr Allergy Immunol. 2013 Aug;24(5):450-5.

5. Leonard SA, Sampson HA, Sicherer SH, Noone S, Moshier EL, Godbold J, Nowak-Węgrzyn A. Dietary baked egg accelerates resolution of egg allergy in children. J Allergy Clin Immunol. 2012 Aug;130(2):473-80.e1.

6. Leonard SA. Debates in allergy medicine: baked milk and egg ingestion accelerates resolution of milk and egg allergy. World Allergy Organ J. 2016 Jan 26;9:1.

7. Fleischer DM, Perry TT, Atkins D, Wood RA, Burks AW, Jones SM, Henning AK, Stablein D, Sampson HA, Sicherer SH. Allergic reactions to foods in preschool-aged children in a prospective observational food allergy study. Pediatrics. 2012;130:25-32.

8. Eigenmann PA, Caubet JC, Zamora SA. Continuing food-avoidance diets after negative food challenges. Pediatr Allergy Immunol 2006; 17(8):601-5.

9. Van der Valk JPM, Gerth van Wijk R, Vergouwe Y, de Jong NW. Failure of introduction of food allergens after negative oral food challenge tests in children. Eur J Pediatr . 2015; 174(8):1093-9.

10. Van Erp FC, Boot J, Knulst AC, Pasmans SGM, Van der Ent CK, Meijer Y. Reintroduction failure after negative peanut challenges in children. Pediatr Allergy Immunol. 2014;25(6):580-5.

\section{Hosted file}

Figure Avoidnace of cooked egg after OIT or OFC .docx available at https://authorea.com/ users/438321/articles/539553-reintroduction-failure-after-undergoing-oral-immunotherapyor-oral-food-challenges-with-cooked-egg 